LETTERS TO THE EDITOR.

[The Editor does not hold himself responsible for opinions expressed by his correspondents. Neither can he undertake to return, or to correspond with the writers of, rejected manuscripts intended for this or any other part of NATURE. No notice is taken of anonymous communications.]

\section{On the Theory of Magneto-ionisation.}

I BEG permission to make a few remarks on the note in NATURE of September 13, p. 32, in which orie of my latest papers is criticised, the one in which 1 gave, I think, a direct proof of magneto-ionisation.

In the experiment which the writer of the note takes into consideration the intensity of the current in the air ionised by a beam of X-rays between two parallel metal plates is measured. I find that a magnetic field directed perpendicularly to the electric field, and the intensity of which is below a certain value, causes an increase of current, although the deviations of the ions and electrons tend to produce a decrease. This effect is uncertain in the case of small potential differences, but it becomes considerable when ionisation by collision begins.

The writer of the note remarked that "when a magnetic field of 430 gauss is superimposed, there is found a current decrease ..." Now, the numerical table to which he alludes is not the only one given in the paper, and it shows such a decrease (which is very small) in one case only, which may be considered as accounted for by experimental error, since nothing of the kind is found in the other numerical tables. That, of course, leads one to suppose that the writer is not fully acquainted with certain parts of my work, and what he says farther on seems to confirm this supposition: "In the opinion of the writer of this note Prof. Righi's interpretation of his results is by no means the only one which is possible, and though his ingenious experiments are of great interest, his theory will need further support before it obtains general acceptance. In particular, it will be necessary to show that the increase of current is not caused by the oblique, and therefore longer, paths of the ions under the joint actions of the two fields."

The writer then offers a new theory, or, more exactly, he states a general idea, which he seems to consider to be preferable to my theory. But this idea cannot be admitted, as I now propose to show.

Apart from the fact that the writer appears to believe that the effect of the magnetic field is simply the obliquity of the trajectory of the ions, whereas these trajectories become certain well-known curves (which may be deduced from the formulæ given in the third paragraph of the note added to my paper), I at once make the fundamental objection that it is not sufficient to increase the distance travelled over by an ion in order that the latter may become capable of ionising a larger number of atoms. In fact, as in ionisation by collision an ion loses a pant of its kinetic energy, it would be necessary to prove that the magnetic field causes the said energy to increase. Now this is not at all the case, since by means of the formulæ of the movement of an ion in an electric and magnetic field it is demonstrated that when the ion traverses a plane perpendicular to the electric field it possesses exactly the same velocity, be the magnetic field existent or not.

It may be added that when the magnetic field does exist, the speed of the ion may increase only to a maximum value, after which it decreases again, the ion retroceding in respect of the lines of electric force, while if the magnetic field does not exist, the velocity mav increase without limit, provided it be not stopped by the electrode which attracts it or by collision with NO. 2508 , VOL. IOO] molecules. All this is clearly understood when one knows that, apart from the velocity parallel to the magnetic force, which remains constant, the trajectory of the ion is nothing but the curve described by a point travelling at a constant speed over a circumference, while the latter is itself travelling uniformly in a straight line in a direction perpendicular to the two fields.

Finally, I wish to point out that, even if the idea expressed by the writer of the note did not lack a basis, my theory could not easily be rejected. It is, in fact, but a direct consequence of the conceptions already accepted by most physicists, nor does it need the addition of any subsidiary hypothesis.

As a matter of fact, it being admitted that atoms. have satellite electrons, they must tend to orient themselves in the magnetic field as if the orbits of such electrons were closed electric currents. Now the sense of this orientation is such that the force due to the field, and acting on the electrons, is directed towards the outside of the orbits, which causes a decrease of the energy required to detach them from the rest of the atoms.

It is this facility of ionisation produced by the magnetic field that constitutes "magneto-ionisation."

Bologna (Italy), September 27 . Augusto Righi.

I AM sorry if, owing perhaps to the brevity of my note, I have led Prof. Righi to think that, in my opinion, his theory of magneto-ionisation can be "easily rejected." Nothing was further from my intention. I believe I have read all Prof. Righi's papers on the subject as they have appeared, and have repeated some of his experiments. The impression they have left on my mind is that, although Prof. Righi's theory gives a plausible explanation of the complex phenomena investigated, yet it is not the only one possible, and further work is necessary before a final conclusion can be reached. I did not express a preference for another theory. I merely suggested objections that would have to be met before the theory under discussion could be unhesitatingly accepted. The question as to whether I have given a fair account of the numerical results must be left to the decision of the readers of the memoir.

THE. Writer OF the NOts.

\section{The Introduction of the Word "Magneton."}

THE word "magneton" is now so frequently used that it has seemed worth while to me to seek to learn to whom is due this addition to the vocabulary of physics.

So far as I can find, Dr. L. A. Bauer was the first to employ the word. In the weekly journal, Science (June Io, I9Io, vol. xxxi., p. 920), is a report of a meeting of the Philosophical Society of Washington, D.C., held on May 7, igro. There is included an abstract of a paper by Dr. Bauer entitled "Is there an Emanation from a Magnetised Substance?" in which the following occurs:- "The corpuscles in magnetism might be atomic systems in which the electron is revolving about an inner nucleus consisting, for example, of a positive ion, such as assumed by Righi for the formation of his so-called 'magnetic rays.' . . . Since the system creates an atomic magnetic field the axis of which passes through the centre of rotation of the electron and perpendicular to the plane of rotation, the speaker suggested calling such systems ' magnetons." "'

In a letter to me Dr. Bauer says:-"The term was used not only in my paper before the Philosophical Society of Washington on May 7, igro, but also in my 